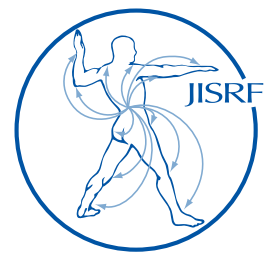

Check for updates

\title{
Modular Necks and Corrosion - Review of Five Cases
}

\author{
McTighe $T^{1}$, Brazil $D^{2}$ \\ Acknowledgment: John Keggi ${ }^{3}$, Louis Keppler ${ }^{3}$, Bradley K. Vaughn ${ }^{3}$, and Edward McPherson ${ }^{3}$
}

\begin{abstract}
This report updates previous articles and commentary presented on Modular Necks from our Tissue Sparing Implant Study Group. In July 2012 we reported that at two years post-op we had encountered no modular neck taper failures or any signs of fretting corrosion, or pseudo tumors associated with the $\mathrm{ARC}^{\mathrm{TM}}$ Stem.

Today we describe five patients out of five hundred and forty-two who had total hip arthroplasty revision [titanium alloy stem, cobalt-chromium modular neck (c.c.) and c.c. modular head (32 $\mathrm{mm}$ or 36 $\mathrm{mm}$ ), highly-cross-linked polyethylene liner, metal titanium plasma sprayed cementless metal cup]. All patients' were female and all demonstrated progressive hip pain or late instability. All had debridement of the periarticular soft tissue, stem extraction with new primary length cementless stem replacement. At revision and early follow up all patients are doing well, however, we recommend heighten awareness in all active female patients with modular neck stem junctions.
\end{abstract}

Keywords: modularity, tapers, corrosion, modular necks

Level of Evidence: AAOS Therapeutic Level IV

\section{Introduction}

Corrosion of metals has many different mechanisms that all have independent driving forces. One such corrosion mechanism that has recently been attributed to the decline in the clinical acceptance of modular-neck hip implants and recall of two products by Stryker Orthopaedics (Mahwah, NJ) is that of fretting corrosion - that is, component damage within the modular connections. [1]

Stem-neck modularity has been under heightening scrutiny since 2012 with the Safety Alert of the Stryker Rejuvenate modular neck-stem implant [2]. This alert was issued within two years of product introduction into the
1. Timothy McTighe, Dr HS (hc); Joint Implant Surgery \& Research Foundation, 46 Chagrin Shopping Plaza, \#117, Chagrin Falls, OH 44022 US

2. Declan Brazil, PhD; Signature Orthopaedics, 7 Sirius Rd Lane Cove West NSW $2066 \mathrm{AU}$

3. Member of the Tissue Sparing Implant Study Group, JISRF (Direct reprint requests to Timothy McTighe)

(C) 2016 McTighe, Brazil. All rights reserved.

Reconstructive Review is a peer-reviewed, open-access orthopaedic oPEN 2 ACCESS journal devoted to publishing papers in the area of reconstructive

arthroplasty. Authors retain copyright and grant the journal right of first publication with the work. Reconstructive Review follows the Creative Commons AttributionNonCommercial CC BY-NC. This license allows anyone to download works, build upon the material, and share them with others for non-commercial purposes as long as they credit the senior author, Reconstructive Review, and the Joint Implant Surgery \& Research Foundation (JISRF). 
USA market. The Safety Alert defines the potential hazards as follows: "Excessive metal debris and/or ion generation. Fretting and/or corrosion at or about the modular neck junction may lead to increased metal ion generation in the surrounding joint space. Contact between metal ions and tissues and structures during an implant's service life may result in an Adverse Local Tissue Reaction (ALTR), the inflammation of associated tissues experiencing immunological response (metallosis, necrosis, and/or pain)[3].

Proximal neck-stem modularity has a long history in the market dating back to the proximal modular body of the McBride Hip in 1948 and was followed in 1978 by Bousquet and Bornand with the development of a proximal modular neck-stem that featured a proximal body that was attached to a stem via a conical mounting post. The BSP Modular Stem followed in 1988 and featured a modular collar/ neck assembly that was fixed to the stem with a Morse taper joint. $[4,5,6]$.

These were early cementless stem designs and not widely used so there is limited clinical reports on these early modular junctions [4]. These modular designs were bulky with matching metallic alloys. To our knowledge there has never been a report of corrosion or modular junction failure with these early bulky modular junctions. Figure 1.

Modern day designs (2000) have been used primarily in conventional cementless titanium alloy stems with either titanium alloy or cobalt-chromium (c.c.) modular necks. The issues that have plagued these modular neck designs have been fatigue failure of both titanium alloy, and c.c. along with fretting corrosion of the cobalt-chromium necks used with the titanium alloy stems [6,7]. Figure $2 \& 3$.

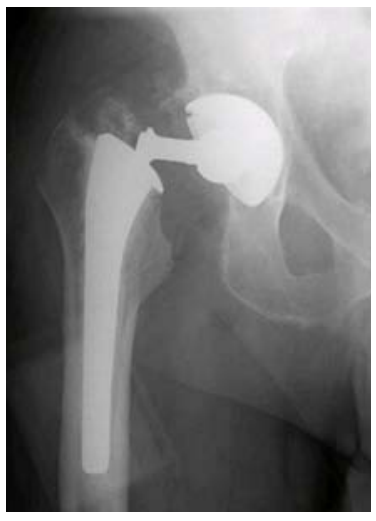

Figure 1. Illustration of Proximal Modular Neck Body 1978 by Bousquet \& Bornand (Courtesy JISRF \& Chris Burgess, Signature Orthopaedics Ltd.)

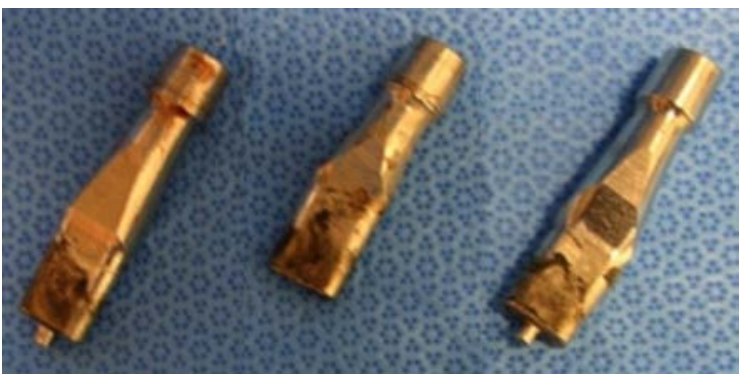

Figure 3. Retrieval of taper corrosion with dissimilar metals-cobalt-chrome alloy modular neck on titanium stem. (Courtesy WL Walter)

The use of modular necks in neck-preserving stem designs has had fewer problems in both areas of fatigue and fretting corrosion as compared to conventional neck resection stems.. The neck-preserving stem reduces both torsional and bending loads reducing the overall stress placed on the modular taper junction. $[7,8,9]$ Figure 4.

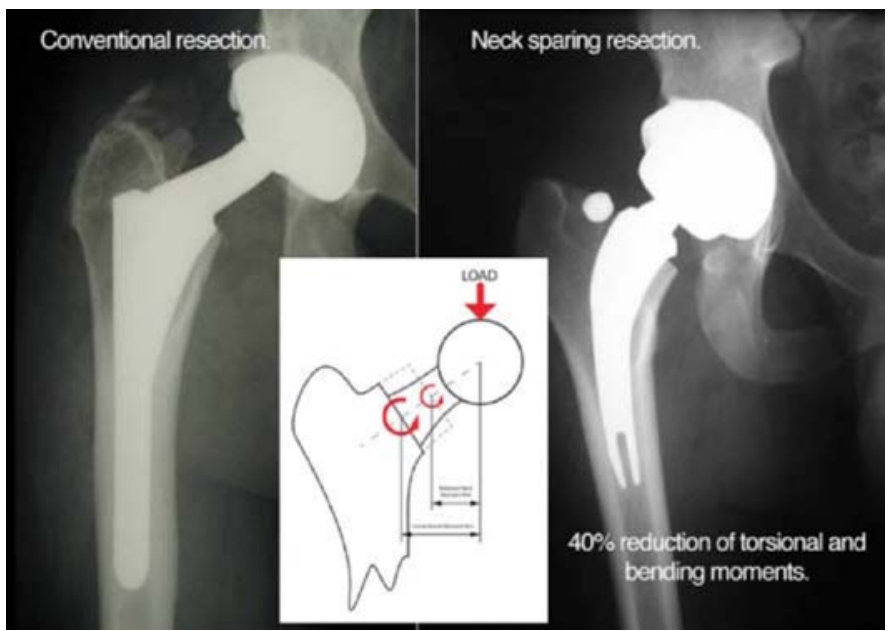

Figure 4. Two Postoperative X-rays showing different level neck resections and offsets resulting in less bending and tosional moments in the neck-sparing implant. (Courtesy of JISRF)

The purpose of this commentary is to report our experience with a short curved neck-preserving cementless stem (titanium alloy) used with a modular neck junction of a different material (c.c.) resulting in suspected corrosion failure of the modular neck-stem junction. Figure 5.

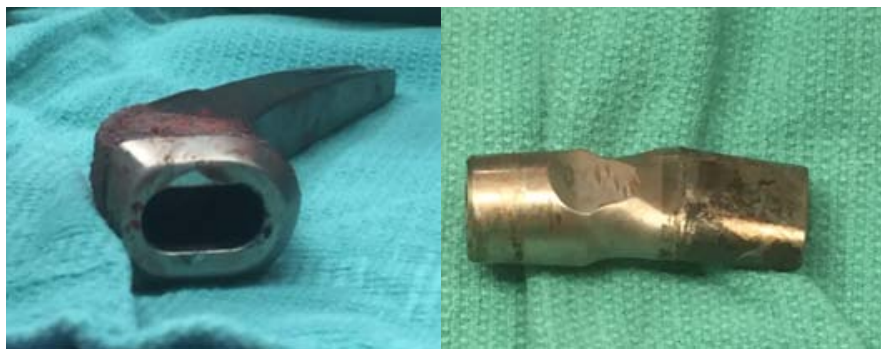

Figure 5. Explanted ARC stem 5 years post-op (Omni, Raynham MA,) Courtesy of B. Vaughn 


\section{Materials and Methods}

This is a case series of five patients treated with primary cementless total hip arthroplasty (THA) between April 2010 and June 2016. Stem was the Omni ARC ${ }^{\text {TM }}$ necksparing stem (titanium alloy stem with hydroxyapatite coating applied to the porous coating and a c.c. modular taper neck) Figure 6.

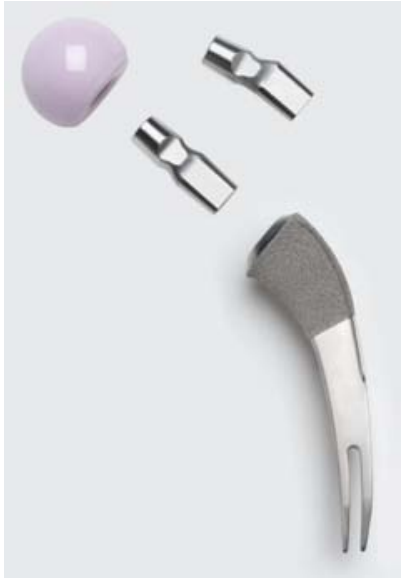

Figure 6. Omni ARC ${ }^{T M}$ Stem (Omni, Raynham MA) Titanium Alloy Stem with $H A$ and pure titanium plasma spray coating with a c.c. Modular neck.

There were 542 primary arthroplasties performed at four centers during this time frame. Revision procedures on these five patients were performed between December 2012 and August 2016.

Patients presented with clinical symptoms for progressive hip pain or late instability. Three of the five patients were tested for serum or blood cobalt and chromium ion levels. Two patients demonstrated increased metal ion levels. One patient had normal levels. The average time between primary THA and revision surgery was 4.6 years (range 3.4-6.2 years). This is similar to previous reports on trunnion corrosion with head-neck modular taper junctions. [10]

All stems were well fixed and revised by indexed surgeons to a contemporary cementless monoblock style stem. In all four cases there was minor discolored staining of implants and surrounding tissue. Figure 7

Figure 7. Tissue and bone debris showing slight staining from corrosion action of modular neck-stem taper. Courtesy of B. Vaughn

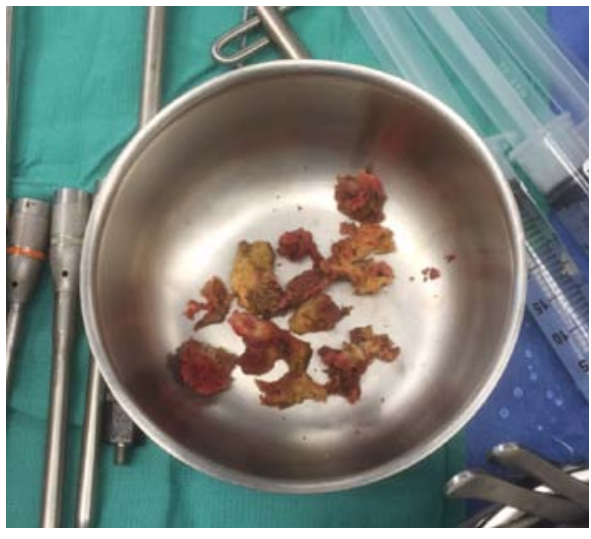

\section{Intraoperative Findings}

All femoral stems were well fixed with minor black staining of surrounding tissue and retrieved modular necks. Four of the modular necks were angled positions with one being neutral. Minor abnormal looking tissue was debrided with no abductor muscle necrosis observed. Figure 8

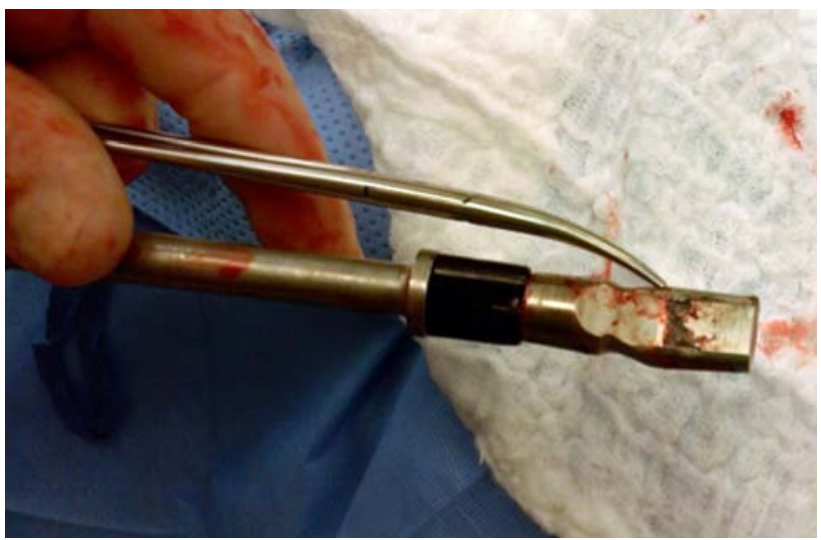

Figure8. Extracted modular neck with black staining. Courtesy L. Keppler

Material analysis was not preformed on the explanted devices. The neck-stem taper junction of each device was found to be well fixed with no signs of gross motion. Instrumentation allowed for ease of stem extraction with minor bone damage and conversion to a cementless primary stem length design. Figure 9.

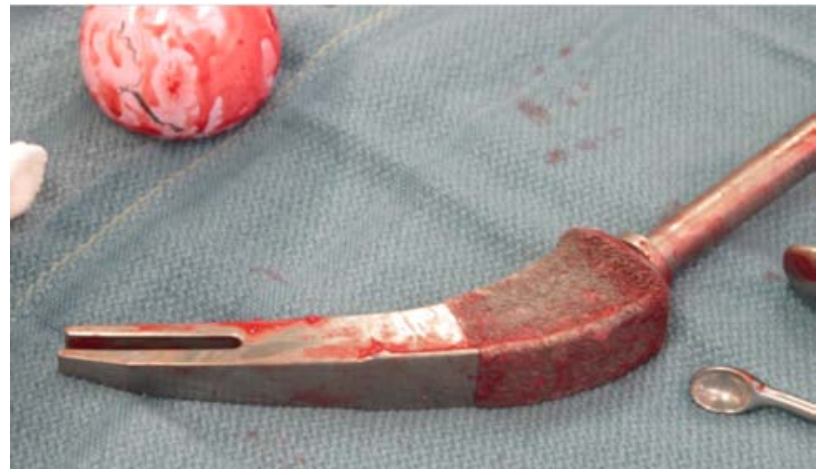

Figure 9. Retrieval instruments allow for ease of extraction. Courtesy JISRF

\section{Discussion}

Corrosion of taper junctions does occur and can result in significant pain and disability for patients. Reports of neck-stem modular junction corrosion in neck-preserving stems is uncommon however our case report of five patients out of five hundred and forty-two (1.0\% revision rate) does demonstrated that there is a risk factor and appears to be more susceptible in active women. Historical publications demonstrate that females respond more fre- 
quently to metal sensitivity than males. [11, 12]

The etiology of neck-stem modular taper junction corrosion in our small series is unclear. Implant design features, neck angle, material, length, offset, head diameter, gender and patient related activities may influence the overall risk of modular junction corrosion. Although we feel the clinical use of modular neck-stem components has performed better in neck-preserving designs as compared to conventional neck resection designs it is obvious that neck-preserving modular neck designs are not without risk of corrosion at the modular junction site.

Ongoing research and testing on enhanced modular neck designs are demonstrating significant reduction in fretting abrasion debris and should be available in the near future. $[1,8]$ We have also seen design improvements in two specific designs that have had identified clinical failure problems and have made design changes that to resolve those problems. [1,13] Figure $10 \& 11$
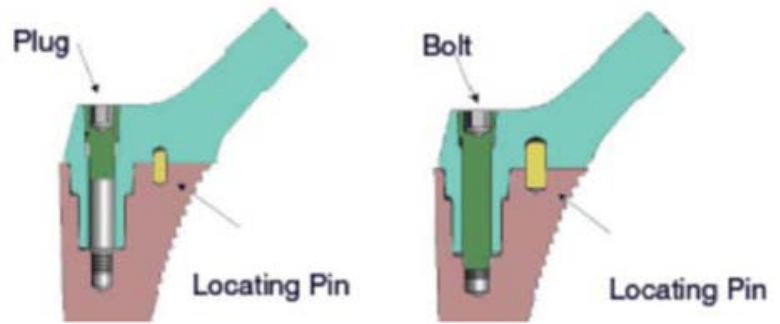

Figure 10. Illustration showing old Dual-Press design to new improved design increasing torsional resistance from $95 \mathrm{ft}$-lbs to $216 \mathrm{ft}-\mathrm{lbs}$.
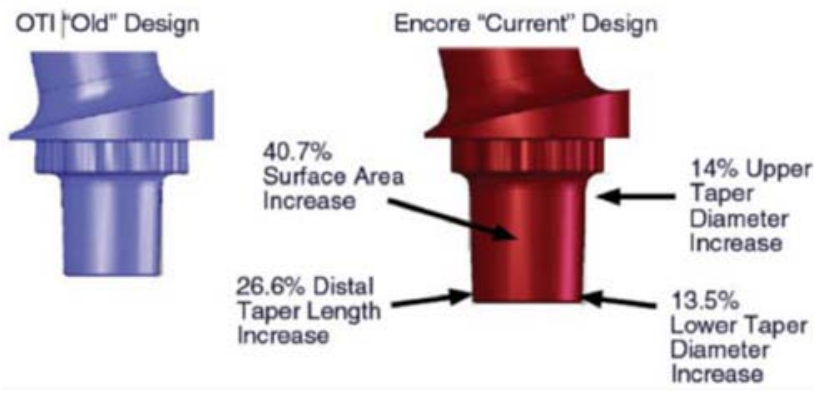

Figure 11. Illustration showing modular taper improvements from the original OTI ${ }^{T M}$ design to the Encore improvement design. (Courtesy of JISRF)

\section{Conclusion}

Modular neck-stem taper junctions are not without risk, however, the potential benefit of fine-tuning joint mechanics (as with head-neck modularity) warrant not only continued use but active research to develop enhanced designs that will reduce or eliminate the current fatigue and corrosion concerns of these modular junctions. The monoblock version of this stem can address many varieties of proxi- mal femoral anatomy. In specific cases where the modular version is required to properly reconstruct hip biomechanics, we recommend heightened awareness of revision risk for highly active females.

\section{Disclosure}

One or more of our authors have disclosed information that may present potential for conflict of interest with this work. For full disclosures refer to last page of this journal.

\section{References}

1. McTighe T, Brazil D, Bruce W. Metallic Alloys in Total Hip Arthroplasty. In: Cashman J, Goyal N, Parvizi J, eds. The Hip: Preservation, Replacement and Revision. Baltimore, MD: Data Trace Publishing Company; 2015:14-1-14-12.

2. Stryker Field Safety Notice RA2012-067 ABGII Modular Stems and ABGII Modular Necks Rejuvenate Modular Stems and Rejuvenate Modular Necks.

3. Gilbert JL, Buckley CA, Jacobs JJ. In vivo corrosion of modular hip prosthesis components in mixed and similar metal combinations. The effect of crevice, stress, motion, and alloy coupling. J Biomed Mater Res. 1993 Dec; 27(12): 1533-44.

4. Bousquet, G., Bornard, F.: "A Screw-Anchored Intramedullary Hip Prosthesis" 1984 Morscher Book pages 242-246 Springer-Verlag Cementless Fixation of Hip Endoprostheses"

5. Keppler L, McTighe T. The Role of Stem Modularity for THA in a Community Based Practice. Reconstructive Review Volume 2(Number 2): 16 August 2012

6. McTighe T, Brazil D. -Memorandum- Modular Necks. Reconstructive Review 2(2) August 2012 DOI: $10.15438 /$ rr.v2i2.22

7. McTighe T, Brazil D, Clarke IC, McPherson E, et al. Metallic Modular Taper Junctions in Total Hip Arthroplasty. Reconstructive Review 5(2) August 2015 DOI: 10.15438/rr.5.2.108

8. McTighe T, Brazil D, Keggi J, Keppler L, et al. Optimizing-Modular-Tapers-forTotal-Hip-Arthroplasty-ICJR-July2014. Conference Paper July 2014: International Congress for Joint Reconstruction, Pacific Hilton Waikoloa Village Resort.

9. McTighe T, Brazil D, Keggi J, Keppler L, et al. Short-Stem Designs for Total Hip Arthroplasty: Neck Stabilized Femoral Components. In Callaghan J, Beaule P, Clohisy J, et al. The Adult Hip. 3rd ed. Philadelphia: Wolters Kluwer Health, in press.

10. Manthe M, Blasser K, Beachamp C, O'Connor MI. Trunnion Corrosion Causing Failure in Metal-on-Polyethylene Total Hip Arthroplasty with Monolithic Femoral Components. Reconstructive Review Vol 6, No 1, April 2016 http://dx.doi. org/10.15438/rr.6.1.131

11. Stejskal V, Danersund A, Lindvall A, et al. Metal-Specific Lymphocytes: Biomarkers of Sensitivity in Man. http://www.melisa.org/pdf/biomark2.pdf

12. McPherson L, McPherson E, McTighe T. Diagnosis \& Management of Hypersensitivity in the Painful THA. Poster Exhibit P128 AAOS 2011 Annual Meeting.

13. Cameron H, McTighe T. Experience with Modular Necks for Cemented Total Hip Arthroplasty. RR Vol 6, No1 April 2016 http://www.melisa.org/pdf/biomark2.pdf 